\title{
Usefulness of Omalizumab and Sting Challenge Test in Hymenoptera Venom Allergy and Mastocytosis
}

González-de-Olano D ${ }^{1,6}$, Padial-Vilchez $\mathrm{MA}^{2}$, Núñez-Acevedo B2, de-Calzada-Bustingorri MP ${ }^{1}$, de-Andrés-Martín $\mathrm{A}^{3}$, Caldas $\mathrm{C}^{4,6}$, Piris-Villaespesa $\mathrm{M}^{5}$, de-la-Hoz-Caballer $\mathrm{B}^{1,6}$

${ }^{1}$ Allergy Department, Hospital Universitario Ramón y Cajal, Madrid, Spain

${ }^{2}$ Allergy Section, Hospital Infanta Sofia, Madrid, Spain

${ }^{3}$ Immunology Department, Hospital Universitario Ramón y Cajal, Madrid, Spain

${ }^{4}$ Sequencing DNA Service (NUCLEUS), University of Salamanca, Salamanca, Spain

${ }^{5}$ Hematology Department, Hospital Universitario Ramón y Cajal, Madrid, Spain

${ }^{6}$ Spanish Network on Mastocytosis (REMA)

J Investig Allergol Clin Immunol 2019; Vol. 29(4): 318-320 doi: 10.18176/jiaci.0395

Key words: Anaphylaxis. Hymenoptera. Mastocytosis. Omalizumab. Sting challenge test.

Palabras clave: Anafilaxia. Himenópteros. Mastocitosis. Omalizumab. Test de repicadura.

A clear relationship has been demonstrated between hymenoptera venom anaphylaxis (HVA) and systemic mastocytosis (SM). In fact, up to $26 \%$ of the patients with a lifethreatening reaction after sting by bees and/or wasps present clonal mast cells (MCs) [1]. Venom immunotherapy (VIT) has proved to be effective in SM patients who have an IgE-mediated reaction, although there is a higher risk of adverse reactions than in the general population, especially in the build-up doses. Consequently, omalizumab may be of use while attempting to achieve maintenance [2]. VIT is effective in most patients treated and in around $75 \%$ of patients with SM [2], although no in vitro techniques are able to predict the effectiveness of treatment in a specific patient [3]. Sting challenge testing (SCT) has proven to be the most reliable method and the gold standard for monitoring the effectiveness of treatment [4]. Nevertheless, its use in patients at higher risk of reactions such as patients with clonal MC disease remains controversial.

A 32-year-old man, who was a beekeeper as a hobby, was stung by 8 honeybees in the summer of 2015 and immediately experienced general malaise, dizziness without loss of consciousness, and chest tightness. The result of the intradermal skin test was positive with Apis mellifera extract at $0.1 \mu \mathrm{g} / \mathrm{mL}$ and negative with Vespula species and Polistes dominula up to a concentration of $1 \mu \mathrm{g} / \mathrm{mL}$ (ALK Allergologisk Laboratorium A/S). Specific IgE (sIgE) determinations (ImmunoCAP, Thermo Fisher and Immulite 2000, Siemens Diagnostics) were carried out and showed positive results (Figure). VIT was initiated without premedication (Pharmalgen Apis mellifera, ALK Allergologisk Laboratorium A/S) and a maintenance dose of $100 \mu \mathrm{g}$ was reached in 3 weeks without 
incident. Within minutes of the seventh monthly maintenance dose (which belonged to the same batch as the previous doses), the patient experienced chest tightness, dizziness, and a rapid drop in blood pressure to $60 / 40 \mathrm{mmHg}$. He was premedicated with $50 \mathrm{mg}$ of oral prednisone and $10 \mathrm{mg}$ of loratadine for the next monthly dose, and the $100-\mu \mathrm{g}$ dose was split between both arms, although he presented the same reaction as in the previous month. MC disease was then suspected. VIT was interrupted and a bone marrow study was performed to evaluate all the disease characteristics used for the diagnosis of $\mathrm{SM}$. The study involved bone marrow MC cytology, histology, immunochemistry, flow cytometry immunophenotyping based on specific gating strategies for the detection of bone marrow MCs present at low frequencies, and the study of the KIT mutation in purified MCs [5]. A final diagnosis of indolent systemic mastocytosis without skin involvement (ISMs-) was reached, and the patient started therapy with disodium cromoglycate daily as part of the recommended treatment for this disease. In order to resume immunotherapy, he began with $300 \mathrm{mg}$ of omalizumab every 4 weeks. After 2 doses, VIT was restarted without additional premedication and with good tolerance. The monoclonal antibody was maintained for a year - a little longer than the time elapsed in previous reactions with VIT — and a basophil activation test was performed before discontinuation, with negative results (Figure). Most patients with mastocytosis are protected by VIT, although a minority may develop systemic adverse reactions after re-exposure. In this situation, it is recommended to increase the maintenance dose to $200 \mathrm{mg}$ or more, since the degree of therapeutic success correlates with the dose of venom [6]. Furthermore, Api $\mathrm{m} 2$ seemed to be the culprit allergen in the present case and has been considered a risk factor for systemic adverse reaction [7]. Given that the patient had not undergone field re-sting since the beginning of VIT, when we confirmed the decrease in sIgE values once the effect of omalizumab had disappeared (9 months, according to the manufacturer), SCT was performed according to the proposed methodology [4] without prompting a reaction.

Although the present study is based on only 1 case report, several relevant conclusions can be drawn. Patients presenting with anaphylaxis to hymenoptera stings are at high risk for clonal MC disorders [1]. Along with the determination of baseline serum tryptase in patients with anaphylaxis following hymenoptera stings, the clinical features of anaphylaxis should also be taken into account, since there is an association between the presence of cardiovascular symptoms in the absence of both urticaria and angioedema and ISMs-- and clonal MC activation syndromes [8]. Furthermore, in ISMs-, MCs represent only a very small proportion of all nucleated bone marrow cells, and serum baseline tryptase levels are usually under reference values $(<11.4 \mu \mathrm{g} / \mathrm{L})$. The diagnosis of $\mathrm{MC}$ diseases requires the performance of a bone marrow biopsy, which is an expensive,

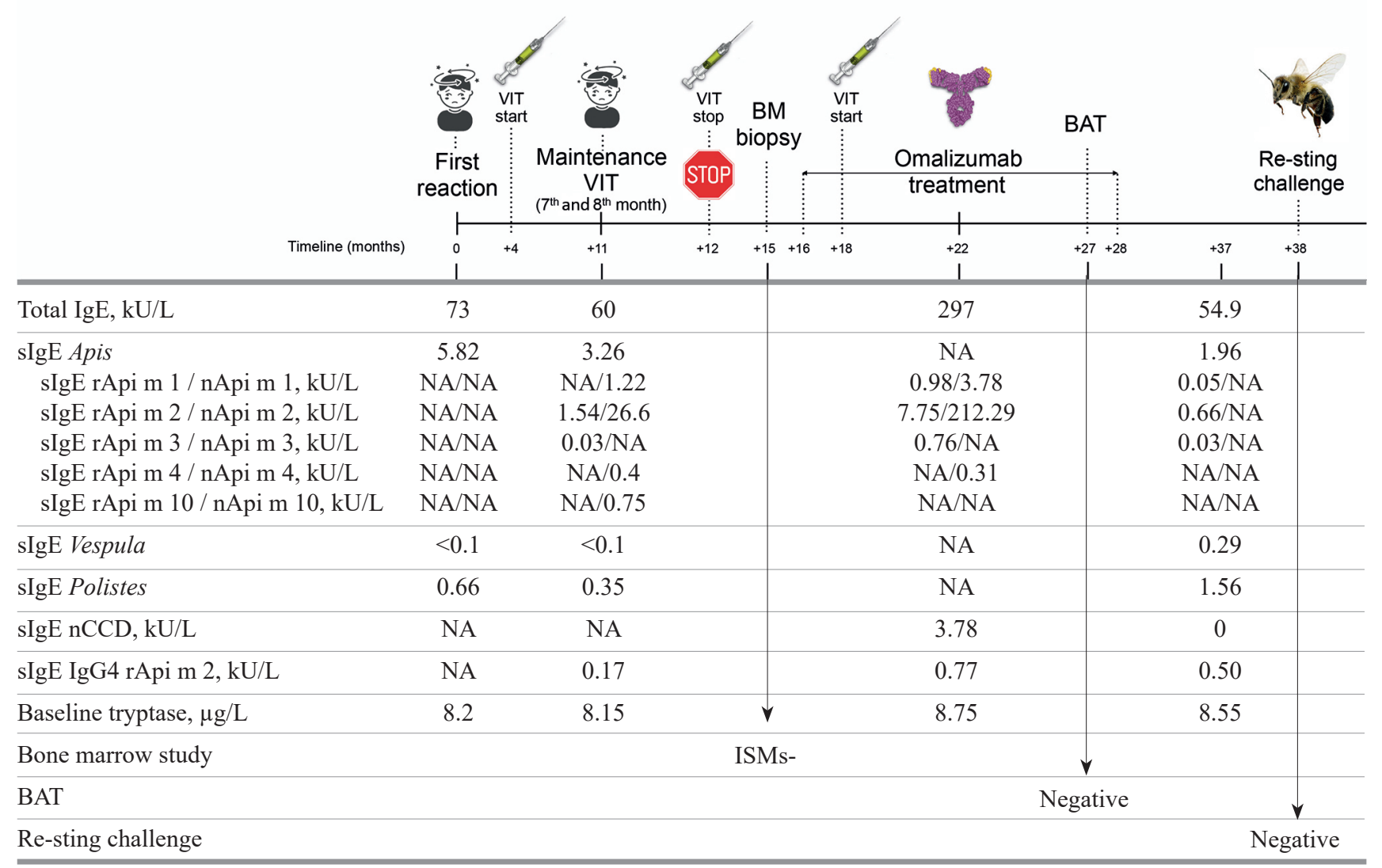

Figure. Chronological summary of the study and allergology work-up. BAT indicates basophil activation test; BM, bone marrow; CCD, cross-reacting carbohydrate determinants; ISMs-, indolent systemic mastocytosis without skin lesions; n, native; NA, not available; r, recombinant; slgE, specific immunoglobulin E; VIT, venom immunotherapy. 
sensitive, and invasive procedure. It is therefore important to define criteria to select patients with HVA who should undergo this procedure [5]. In this context, screening tools that help in predicting clonality with high sensitivity and specificity, such as the REMA Score, are useful [8]. Another relevant issue is the usefulness of component-resolved diagnosis (native or recombinant) to facilitate identification of the allergen responsible for a reaction, especially when the culprit allergen might be associated with a higher rate of systemic adverse reactions or might be underrepresented in commercial diagnostic or therapeutic extracts. Moreover, omalizumab has demonstrated its utility as a VIT adjuvant in patients with clonal MCs who were unable to tolerate immunotherapy [2]. The potential role of disodium cromoglycate as a VIT adjuvant remains unknown, although it cannot be excluded. Finally, performing SCT in patients with mastocytosis is a controversial topic, since systemic reactions have been observed in higher rates than in the general population $(23.9 \%$ vs $9.3 \%)$ [9]. Component-resolved diagnosis results in more accurate knowledge of the patient's sensitization profile, which might help to decrease the rate of systemic reactions. Nevertheless, this assumption needs further assessment. SCT has shown the highest correlation for evaluation of the effectiveness of VIT and for improving health-related quality of life [4], and patients who react to a sting challenge while receiving conventional VIT are protected by an increased maintenance dose [10]. Mastocytosis is known to be closely related to HVA [2]. Since patients are more likely to present adverse reactions during VIT and are at greater risk of recurrence once treatment is discontinued, experts recommend that they should receive lifetime VIT $[3,4]$. Therefore, an extremely cautious approach would involve performance of SCT, if needed, in a patient with clonal MCs and an accurate diagnosis based on molecular sensitization patterns.

\section{Funding}

The authors declare that no funding was received for the present study.

\section{Conflicts of Interest}

David González-de-Olano belongs to the Spanish Thematic Network and Co-operative Research Centres ARADyAL, Center RD16/0006/0022. Belén de-la-Hoz-Caballer belongs to the Spanish Thematic Network and Co-operative Research Centres ARADyAL, Center RD16/0006/0009. The remaining authors declare that they have no conflicts of interest.

\section{References}

1. Vázquez-Revuelta P, González-de-Olano D. Prevalence of Clonal Mast Cell Disorders in Patients Presenting With Hymenoptera Venom Anaphylaxis Might Be Higher Than Expected. J Investig Allergol Clin Immunol. 2018;28:193-4.

2. González-de-Olano D, Alvarez-Twose I, Vega A, Orfao A, Escribano L. Venom immunotherapy in patients with mastocytosis and hymenoptera venom anaphylaxis. Immunotherapy. 2011;3:637-51.
3. Alfaya Arias $T$, Soriano Gómis $V$, Soto Mera $T$, Vega Castro $A$, Vega Gutiérrez JM, Alonso Llamazares A. et al. (Hymenoptera Allergy Committee of the SEAIC). Key Issues in Hymenoptera Venom Allergy: An Update. J Investig Allergol Clin Immunol. 2017;27:19-31.

4. Sturm GJ, Varga EM, Roberts G, Mosbech $H$, Bilò MB, Akdis $C A$, et al. EAACl guidelines on allergen immunotherapy: Hymenoptera venom allergy. Allergy. 2018;73:744-64.

5. González-de-Olano D, Matito A, Orfao A, Escribano L. Advances in the understanding and clinical management of mastocytosis and clonal mast cell activation syndromes. F1000Res. 2016 Nov 14;5:2666.

6. Ruëff F, Vos B, Oude Elberink J, Bender A, Chatelain R, DugasBreit $S$, et al. Predictors of clinical effectiveness of Hymenoptera venom immunotherapy. Clin Exp Allergy. 2014;44:736-46.

7. Ruiz B, Serrano P, Verdú M, Moreno C. Sensitization to Api m 1, Api m 2, and Api m 4: association with safety of bee venom immunotherapy. Ann Allergy Asthma Immunol. 2015;114:350-2.

8. Alvarez-Twose I, González de Olano D, Sánchez-Muñoz L, Matito A, Esteban-López MI, Vega A, et al. Clinical, biological, and molecular characteristics of clonal mast cell disorders presenting with systemic mast cell activation symptoms. J Allergy Clin Immunol. 2010;125:1269-78.e2.

9. Niedoszytko M, de Monchy J, van Doormaal JJ, Hassem E, Oude Elberink JN. Mastocytosis and insect venom allergy: diagnosis, safety and efficacy of venom immunotherapy. Allergy. 2009;64:1237-45.

10. Ruëff $F$, Wenderoth A, Przybilla B. Patients still reacting to a sting challenge while receiving conventional Hymenoptera venom immunotherapy are protected by increased venom doses. J Allergy Clin Immunol. 2001;108:1027-32.

Manuscript received January 23, 2019; accepted for publication March 29, 2019.

David González-de-Olano Hospital Universitario Ramón y Cajal Servicio de Alergia Cta. Colmenar Viejo km 9,1 28034 Madrid, Spain E-mail: dgolano@yahoo.es 